\title{
NO CENÁRIO ATUAL, QUAL O PAPEL DOS BETABLOQUEADORES NO TRATAMENTO DA HIPERTENSÃO?
}

\author{
IN THE CURRENT SCENARIO, WHAT IS THE ROLE OF BETA-BLOCKERS IN THE \\ TREATMENT OF HYPERTENSION?
}

Franciellen Bruschi Almonfrey', Mariana Bellaguarda de Castro Sepulvida', Roberto Dischinger Miranda

\section{RESUMO}

As teorias sobre a existência de receptores adrenérgicos foram descritas na literatura em meados da década de 40, sendo reconhecido anos depois que a inibição do sistema nervoso simpático por um determinado composto bloqueador beta-adrenérgico poderia beneficiar pacientes com arritmias cardíacas e angina pectoris. Nasceram aí as primeiras substâncias bloqueadoras beta adrenérgicas não seletivas, destacando-se o propranolol. Desde então, centenas de bloqueadores beta adrenérgicos (BB) foram sintetizados e dezenas estão disponíveis em todo o mundo para uso clínico. Os mecanismos farmacológicos dos BBs são múltiplos e variam principalmente de acordo o predomínio de ação nos receptores adrenérgicos e com a lipossolubilidade, entre outros. De maneira geral, ligam-se e produzem um antagonismo competitivo e reversível nos receptores distribuídos pelo organismo. Os principais mecanismos fisiopatológicos propostos para explicar a ação medicamentosa dos BBs na hipertensão arterial sistêmica são redução do débito cardíaco, da resistência vascular periférica, do tônus vasomotor e do volume plasmático, inibição de renina, efeitos no sistema nervoso central, melhoria na adesão vascular e redefinição dos níveis de barorreceptores. Atualmente, os BBs não são preconizados como primeira linha de tratamento de hipertensão arterial sem complicações, porém eles são particularmente úteis para o tratamento da hipertensão na presença de algumas comorbidades não cardiovasculares, como a enxaqueca e o tremor essencial. Além disso, os BBs ganham destaque especial em situações cardiovasculares específicas, como angina sintomática, taquiarritmias, pós infarto agudo do miocárdio, insuficiência cardíaca com fração de ejeção reduzida e como alternativa aos inibidores da ECA ou BRA em mulheres hipertensas mais jovens que planejam engravidar. Vale ressaltar que em algumas destas situações os BBs passam a ter indicação mandatória, mesmo que não associadas à hipertensão arterial.

Descritores: Farmacologia; Bloqueadores Beta Adrenérgicos; Anti-hipertensivos; Hipertensão Arterial Sistêmica; Indicações Terapêuticas.

\begin{abstract}
Theories about the existence of adrenergic receptors were described in the mid-40s, being recognized years later that inhibition of the sympathetic nervous system by a beta-adrenergic blocking compound could benefit patients with cardiac arrhythmias and angina pectoris. That's how the first non-selective beta-blocking adrenergic substances appeared, highlighting propranolol. Since then, hundreds of beta-adrenergic blockers have been synthesized and dozens are available worldwide for clinical use. The pharmacological mechanisms of BBs are multiple and vary mainly according to the predominance of action in adrenergic receptors and with liposolubility. In general, they bind and produce a competitive and reversible antagonism in the receptors distributed by the organism. The main pathophysiological mechanisms proposed to explain the action of BBs in systemic arterial hypertension are reduced cardiac output, peripheral vascular resistance, vasomotor tone and plasma volume, renin inhibition, effects on the central nervous system, improvement in vascular adherence and redefinition of baroreceptor levels. Currently, BBs are not recommended as the first line of treatment for non-complicated arterial hypertension, but they are particularly useful for the treatment of hypertension in the presence of some comorbidities, such as migraine and essential tremor. Besides that, $B B s$ gain special prominence in specific cardiovascular situations, such as symptomatic angina, tachyarrhythmias, post-acute myocardial infarction, heart failure with reduced ejection fraction and as an alternative to ACE or ARB inhibitors in younger hypertensive women planning to become pregnant. It is worth mentioning that in some of these situations BBs are mandatory, even if not associated with arterial hypertension.
\end{abstract}

Keywords: Pharmacology; Adrenergic beta-Antagonists; Antihypertensives; Systemic Arterial Hypertension; Therapeutic Uses.

1 Serviço de Doenças Cardiovasculares, Disciplina de Geriatria e Gerontologia, Escola Paulista de Medicina da Universidade Federal de São Paulo. 


\section{INTRODUÇÃO}

As teorias sobre a existência de receptores adrenérgicos foram descritas na literatura em meados da década de 40 por Ahlquist $^{1}$ e estabelecidas na comunidade científica após o desenvolvimento do dicloro isoprotereno por Powell e Slater em 1958. ${ }^{2}$ Alguns anos depois, James Black reconheceu que a inibição do sistema nervoso simpático por um determinado composto bloqueador beta adrenérgico poderia beneficiar pacientes com arritmias cardíacas e angina pectoris. Foi aí que nasceram as primeiras substâncias bloqueadoras beta adrenérgicas não seletivas, o pronetalol e o propranolol, sendo que este tornou-se medicamento protótipo para uso clínico precoce em pacientes com angina, arritmias e feocromocitoma..$^{3-5}$

O desenvolvimento e a aplicação clínica desta classe farmacológica representam avanços fundamentais na farmacoterapia ${ }^{4}$ e garantiram o prêmio Nobel em medicina e fisiologia a Black em 1988. Desde então, centenas de bloqueadores beta adrenérgicos foram sintetizados e dezenas estão disponíveis em todo o mundo para uso clínico. ${ }^{5} \mathrm{Ne}$ nhuma outra classe de drogas sintéticas demonstrou tamanha utilidade terapêutica, tanto para doenças cardiovasculares quanto para outras doenças. ${ }^{5}$

Em 1977 foi publicado o primeiro relatório do comitê nacional conjunto de detecção, avaliação e tratamento da pressão arterial sistêmica. Na ocasião, o propranolol foi indicado como fármaco de segunda escolha para o tratamento da hipertensão arterial sistêmica (HAS), ${ }^{6}$ seguido dos diuréticos tiazídicos.

Com o desenvolvimento de outros betabloqueadores (BBs) com características farmacológicas que facilitariam a posologia e selecionariam a ação em receptores específicos, a classe tornou-se uma das mais prescritas para essa finalidade até o surgimento dos bloqueadores de canal de cálcio (BCCs) e dos inibidores da enzima conversora de angiotensina (IECAs) na gama de anti-hipertensivos. ${ }^{7}$ Diversos estudos demonstraram que estas classes teriam maior benefício como primeira escolha nesse contexto. Ainda assim, os betabloqueadores proporcionaram um grande legado clínico até o momento e são fundamentais no arsenal terapêutico para o manejo da HAS.

\section{FARMACOLOGIA: UMA CLASSE HETEROGÊNEA}

Os mecanismos farmacológicos dos BBs são múltiplos e variam de acordo com o predomínio de ação nos receptores beta adrenérgicos..$^{5,9}$ De maneira geral, ligam-se e produzem um antagonismo competitivo e reversível nos receptores distribuídos pelo organismo. Receptores beta 1 são predominantemente presentes nas células cardíacas, enquanto os beta 2 são encontrados também em outros tecidos. Seus efeitos farmacológicos podem ser explicados a partir do conhecimento da atividade do tônus simpático e das respostas desencadeadas por esses receptores nos vários tecidos (Quadro 1). Dessa forma, sua ação é evidente quando o sistema nervoso simpático é ativado, como durante o exercício ou o estresse. ${ }^{9}$

Os BBs podem ser classificados em não seletivos, que atuam bloqueando tanto os receptores beta 1 ( $\beta$ 1) como os beta 2 ( $\beta$ 2) adrenérgicos; e em seletivos, ou seja, que tem efeito superior nos receptores beta 1 , especialmente em doses mais baixas. (Quadro 2)

\section{Farmacocinética}

A diferença na estrutura química de cada betabloqueador gera diferenças na absorção gastrointestinal, metabolismo hepático de primeira passagem, solubilidade lipídica, ligação a proteínas, distribuição tecidual, concentração cardíaca, taxa de biotransformação hepática e depuração renal, o que influencia na utilização clínica de cada betabloqueador. ${ }^{6}$ Fármacos lipofílicos são completamente absorvidos e metabolizados pelo trato gastrointestinal, atravessam a barreira hematoencefálica e tem meia-vida reduzida quando comparados com os hidrofílicos,

Quadro 1. Efeitos mediados pelos receptores $\beta 1$ e $\beta 2$-adrenérgicos.

\begin{tabular}{|l|l|l|}
\multicolumn{1}{c|}{ Tecido } & Receptor & \\
\hline Coração & & \\
\hline Nó SA & $\beta 1 . \beta 2$ & Aumento da frequência cardíaca \\
Nó AV & $\beta 1, \beta 2$ & Aumento da velocidade de condução \\
Átrios & $\beta 1, \beta 2$ & Aumento da contratilidade \\
Ventrículos & $\beta 1, \beta 2$ & Aumento da contratilidade, velocidade de condução e automatismo do marcapasso ideoventricular \\
\hline Artérias & $\beta 2$ & Vasodilatação \\
Veias & $\beta 2$ & Vasodilatação \\
Músculo esquelético & $\beta 2$ & Aumento de contratilidade, glicogenólise e absorção de potássio \\
\hline Fígado & $\beta 2$ & Glicogenólise e gliconeogênese \\
Pâncreas (células beta) & $\beta 2$ & Secreção de insulina e glucagon \\
Células de gordura & $\beta 1$ & Lipólise \\
Brônquios & $\beta 2$ & Broncodilatação \\
Rim & $\beta 1$ & Liberação de renina \\
Vesícula biliar e ductos & $\beta 2$ & Relaxamento \\
Detrusor da Bexiga & $\beta 2$ & Relaxamento \\
Útero & $\beta 2$ & Relaxamento \\
Terminações nervosas & $\beta 2$ & Liberação de noradrenalina \\
Glândulas paratireoides & $\beta 1, \beta 2$ & Secreção de paratormônio \\
Glândula Tireoide & $\beta 2$ & Conversão de T4 em T3 \\
\hline SA: Sino atrial; AV: Átrio Ventricular. Adaptado de ESC Expert Consensus Document.9
\end{tabular}


Quadro 2. Classificação farmacológica dos betabloqueadores.

\begin{tabular}{|c|c|c|c|c|c|}
\hline Nome & ASI & Lipossolubilidade & $\begin{array}{l}\text { Vasodilatação } \\
\text { Periférica }\end{array}$ & Dose oral média diária & E.V. \\
\hline \multicolumn{6}{|c|}{ I. Antagonistas adrenérgicos não seletivos $\beta 1$ e $\beta 2$} \\
\hline Nadolol* & 0 & Baixa & 0 & $\begin{array}{l}40-320 \mathrm{mg} \\
1 \mathrm{vez} / \mathrm{dia}\end{array}$ & \\
\hline Pindolol & ++ & Alta & 0 & $\begin{array}{l}10-40 \mathrm{mg} \\
2 \text { vezes/dia }\end{array}$ & \\
\hline Propanolol & 0 & Alta & 0 & $\begin{array}{l}40-180 \mathrm{mg} \\
2 \text { vezes/ dia }\end{array}$ & + \\
\hline Sotalol & 0 & Baixa & 0 & & + \\
\hline Timolol & 0 & Alta & 0 & $\begin{array}{l}5-40 \mathrm{mg} \\
2 \text { vezes/dia }\end{array}$ & \\
\hline \multicolumn{6}{|c|}{ II Antagonistas seletivos $\beta 1$-adrenérgicos } \\
\hline Atenolol & 0 & Baixa & 0 & $\begin{array}{l}25-100 \mathrm{mg} \\
1 \mathrm{vez} / \mathrm{dia}\end{array}$ & + \\
\hline Bisoprolol & 0 & Moderada & 0 & $\begin{array}{l}2,5-10 \mathrm{mg} \\
1 \mathrm{vez} / \mathrm{dia}\end{array}$ & \\
\hline Esmolol & 0 & Baixa & 0 & Apenas E.V. & + \\
\hline Metoprolol & 0 & Alta & 0 & $\begin{array}{l}50-100 \mathrm{mg} \\
1 \text { ou } 2 \text { vezes/dia }\end{array}$ & + \\
\hline Nebivolol & 0 & & + & $\begin{array}{l}2,5-5 \mathrm{mg} \\
1 \mathrm{vez} / \mathrm{dia}\end{array}$ & \\
\hline \multicolumn{6}{|c|}{ III Antagonistas adrenérgicos $\beta$ e $\alpha 1$} \\
\hline Carteolol* & + & Moderada & + & $\begin{array}{l}25-100 \mathrm{mg} \\
2 \text { vezes/dia }\end{array}$ & \\
\hline Nadolol* & 0 & Moderada & + & $\begin{array}{l}3,125-50 \mathrm{mg} \\
2 \text { vezes/dia }\end{array}$ & \\
\hline Penbutolol* & + & Baixa & + & $\begin{array}{l}200-800 \mathrm{mg} \\
2 \text { vezes/dia }\end{array}$ & \\
\hline
\end{tabular}

ASI: atividade simpaticomimética intrínseca; E.V.: Administração endovenosa possível; ${ }^{*}$ Indisponível no Brasil. Incluíram apenas betabloqueadores com eficácia demonstrada nos resultados clínicos e apoiando as recomendações das diretrizes. Adaptada de ESC Expert Consensus Document. ${ }^{9}$

que dependem da eliminação renal e não interagem com outros medicamentos de metabolização hepática. A depender do objetivo e do indivíduo a ser tratado, tais características são fundamentais para a escolha do melhor fármaco.

\section{Ação Anti-hipertensiva dos Betabloqueadores}

O Quadro 3 resume os principais mecanismos fisiopatológicos propostos para explicar a ação medicamentosa dos BBs na Hipertensão Arterial Sistêmica.

A redução da frequência cardíaca e da contratilidade do miocárdio com betabloqueadores resulta na diminuição do débito cardíaco o que, a curto e a longo prazo, pode reduzir a pressão arterial especialmente no tratamento da HAS relacionada ao alto débito cardíaco e ao aumento do tônus simpático. ${ }^{6,10}$

Para obter benefício anti-hipertensivo com a redução da resistência periférica, os BBs não seletivos são ineficazes e podem até mesmo predispor mecanismos alfa adrenérgicos por bloquear os receptores de ação opositora. O efeito vasodilatador é mediado por receptores beta 2 no músculo esquelético dos vasos sanguíneos, sugerindo possíveis vantagens terapêuticas no uso de bloqueadores beta 1 seletivos, agentes com atividade agonista parcial e medicamentos com atividade bloqueadora alfa e efeitos vasodilatadores diretos.
Quadro 3. Mecanismos propostos para explicar as ações anti-hipertensivas dos betabloqueadores.

\begin{tabular}{|l|}
\hline 1 Redução do débito cardíaco \\
\hline 2 Inibição de renina \\
\hline 3 Efeitos no sistema nervoso central \\
\hline $\begin{array}{l}4 \text { Efeitos nos receptores beta pré-funcionais: reduções na } \\
\text { liberação de noradrenalina }\end{array}$ \\
\hline 5 Redução da resistência vascular periférica \\
\hline 6 Melhoria na adesão vascular \\
\hline 7 Redução do tônus vasomotor \\
\hline 8 Redução no volume plasmático \\
\hline 9 Redefinição dos níveis de barorreceptores \\
\hline $\begin{array}{l}10 \text { Atenuação da resposta pressora às catecolaminas com exercício } \\
\text { e estresse }\end{array}$ \\
\hline
\end{tabular}

Adaptado de Frishman WH, $2011 .^{6}$ 
Como a seletividade $\beta 1$ diminui à medida que a dose é aumentada e os pacientes hipertensos geralmente necessitam de doses altas de BB, a seletividade $\beta 1$ oferece pouca ou nenhuma vantagem específica no tratamento da hipertensão. ${ }^{6}$

Acredita-se também que o efeito nos receptores pré-juncionais promova redução na quantidade de noradrenalina liberada pelas fibras simpáticas pós-ganglionares, produzindo uma estimulação mais fraca dos receptores pós-juncionais, o que reduziria a vasoconstrição periférica e a PA. ${ }^{6}$

Paradoxalmente ao efeito antagonista, alguns betabloqueadores podem exercer uma resposta agonista fraca denominada atividade simpaticomimética intrínseca (ASI) e, desse modo, estimular e inibir o receptor beta adrenérgico. Além disso, podem apresentar atividade vasodilatadora periférica mediada por bloqueio de adrenorreceptor a1, agonismo do receptor adrenérgico $\beta 2$ ou por mecanismos independentes do bloqueio de adrenorreceptor, ${ }^{9}$ como o carvedilol e o labetalol. Betabloqueadores mais modernos, como nebivolol, apresentam ação vasodilatadora também em decorrência do aumento da liberação de óxido nítrico. ${ }^{11}$

A renina plasmática constitui papel fundamental na regulação da pressão arterial. Acredita-se que os BBs suprimam os níveis plasmáticos da atividade da renina e da angiotensina II, além de reduzir aldosterona urinária em indivíduos normotensos e hipertensos. ${ }^{12}$ Por sua vez, seu nível sérico e efeitos no organismo são decorrentes de fatores distintos como o balanço de sódio, a postura corporal e a pressão de perfusão renal. ${ }^{6}$

Estudo em animais sugerem que o efeito do BB no sistema nervoso central (SNC) contribuiria para a redução da PA, especialmente no caso de drogas lipofílicas que atravessam a barreira hematoencefálica e concentram-se no $\mathrm{SNC}_{1}{ }^{13}$ porém não há evidências clínicas de que exista um efeito anti-hipertensivo direto mediado por sua presença, uma vez que aqueles menos lipossolúveis parecem ser igualmente eficazes no controle da pressão arterial. ${ }^{6}$ Por sua vez, efeitos adversos como cefaleia, distúrbios do sono e transtorno depressivo são menos comuns nas drogas hidrossolúveis. Um dos mecanismos propostos que contribuem para o controle da PA é baseado no efeito ansiolítico dos BBs que reduziria o efeito adrenérgico central e periférico gerado pelo estresse, porém não há estudos robustos que comprovem o mecanismo fisiopatológico dessa ação e independentemente da concentração do fármaco no SNC esse efeito é observado. ${ }^{14}$

Outros efeitos pouco documentados dos betabloqueadores que podem contribuir para suas ações anti-hipertensivas incluem efeitos no tônus venoso e volume plasmático, atividade estabilizadora de membrana e redefinição de barorreceptores. Polimorfismo genético dos receptores $\beta 1$ e $\beta 2$ e outros marcadores genéticos têm sido implicados como causa da hipertensão arterial sistêmica e na capacidade de resposta dos pacientes ao tratamento com betabloqueadores. ${ }^{15,16}$

\section{EVIDÊNCIAS DO USO DE BETABLOQUEADORES NA HAS}

Atualmente os BBs não são preconizados como primeira linha de tratamento de HAS, exceto em situações específicas. ${ }^{17-19}$
Desde a publicação do estudo LIFE em 2002, ${ }^{20}$ diversas metanálises e ensaios clínicos subsequentes revolucionaram a recomendação terapêutica dos betabloqueadores para hipertensão arterial sistêmica. Eles demonstraram que os BB seriam inferiores aos outros agentes anti-hipertensivos na redução de acidente vascular cerebral (AVC) e mortalidade por causas cardíacas e por todas as causas, apesar de reduzirem substancialmente a pressão arterial. ${ }^{20-26}$ O atenolol foi o fármaco mais utilizado nos ensaios clínicos, aproximadamente $75 \%$, e uma pequena fração deles incluiu outros agentes, tais como o propanolol e o pindolol. O impacto dos betabloqueadores de terceira geração (nebivolol e carvedilol) nos resultados clínicos não foram avaliados nos estudos de longo prazo em hipertensão primária ${ }^{8-26}$ e a representação desproporcional do atenolol nessas análises sustenta a dúvidas e a falta de benefício era um efeito de classe ou se estava limitada ao atenolol. ${ }^{27}$

Nos últimos cinco anos, os ensaios clínicos randomizados (ECR) e as metanálises demonstram que, quando comparados ao placebo, os betabloqueadores reduzem significativamente o risco de acidente vascular cerebral, insuficiência cardíaca e eventos cardiovasculares maiores em pacientes hipertensos. ${ }^{28}$ Quando comparados com outros anti-hipertensivos, foram equivalentes na prevenção de eventos cardiovasculares, exceto na prevenção de AVC. .9-31 $^{29}$ Além disso, os ECRs baseados em lesão de órgão-alvo por HAS também indicaram que os betabloqueadores são discretamente menos eficazes que os BCCs e BRAs na prevenção ou regressão da HVE, espessamento intimal carotídeo, rigidez aórtica e remodelamento de pequenas artérias, além de um benefício de mortalidade pós-infarto do miocárdio incerto em pacientes sem disfunção do VE. ${ }^{18,32}$

Desse modo, baseados nestes diversos estudos, os BBs não são mais considerados como terapia inicial preferida para o tratamento da hipertensão arterial essencial. ${ }^{33}$ Eles são particularmente úteis para o tratamento da hipertensão em situações específicas como angina sintomática, taquiarritmias, pós IAM, IC com fração de ejeção reduzida e como alternativa aos inibidores da ECA ou BRA em mulheres hipertensas mais jovens que planejam engravidar. ${ }^{18}$ As principais diretrizes internacionais e a sétima Diretriz Brasileira de Hipertensão endossam e também recomendam que os BBs podem ser considerados fármaco de primeira escolha em situações específicas, como na associação com arritmias supraventriculares, enxaqueca, IC ou coronariopatia. ${ }^{17,18,34}$

Entre o grande número de ensaios clínicos randomizados, apenas alguns compararam diretamente diferentes combinações de dois medicamentos, com o uso sistemático das duas combinações em ambos os braços. Com essa ressalva, nos ensaios que compararam diferentes regimes, não houve grande diferença entre as combinações. Contudo, assim como na monoterapia, os betabloqueadores em combinação devem ser preferencialmente usados quando há uma indicação clínica específica para a sua utilização. Eles podem ser usado sem combinação com IECA/BRA, BCC ou diurético. ${ }^{18}$ 


\section{BETABLOQUEADORES NA HIPERTENSÃO ARTERIAL SISTÊMICA ASSOCIADA A OUTRAS CONDIÇÕES CLÍNICAS}

\section{Doença Arterial Coronariana}

Existe uma forte interação epidemiológica entre Doença Arterial Coronariana (DAC) e hipertensão, responsável por $25 \%$ a $30 \%$ dos infartos agudos do miocárdio. ${ }^{35}$ Além de mudanças no estilo de vida, em pacientes com ambas as comorbidades associadas, BBs e IECA/BRAs melhoram os resultados pós infarto do miocárdio. Do mesmo modo, naqueles com angina sintomática, betabloqueadores e BCC são agente anti-hipertensivos preferidos na estratégia medicamentosa. ${ }^{19,8}$

Diante do exposto, conclui-se que os BBs, independentemente dos níveis pressóricos, com ou sem BCCs, são medicamentos de primeira linha em pacientes hipertensos e coronariopatas. ${ }^{18,36}$

\section{Insuficiência Cardíaca}

A hipertensão é o principal fator de risco para o desenvolvimento de insuficiência cardíaca (IC), e a maioria dos pacientes com IC apresenta histórico de HAS. Ela também causa Hipertrofia Ventricular Esquerda (HVE), que prejudica o relaxamento do ventrículo esquerdo(VE) e é um potente preditor de insuficiência cardíaca, mesmo quando a função sistólica do VE é normal e não há IAM prévio. ${ }^{18} \mathrm{O}$ tratamento da hipertensão tem um grande impacto na redução do risco de IC e hospitalização por tal causa, especialmente em pacientes idosos. ${ }^{37,38}$

Na IC com fração de ejeção reduzida (ICFEr) estabelecida os BBs, especificamente bisoprolol, metoprolol e carvedilol, possuem indicação imperativa, salvo contraindicação absoluta, pela alta eficácia na melhoria dos sintomas e na mortalidade. Por outro lado, não há evidência fortes quanto ao uso de BBs para IC com fração de ejeção preservada. ${ }^{18,34}$

\section{Fibrilação Atrial e outras arritmias}

A hipertensão predispõe a arritmias cardíacas, incluindo arritmias ventriculares, mas mais comumente FA. Mesmo naqueles hipertensos controlados a incidência de FA é elevada e a maioria dos pacientes apresenta alta frequência ventricular, sendo indicado nesses casos betabloqueadores ou antagonistas de cálcio não diidropiridínicos como agentes anti-hipertensivos. ${ }^{18}$

Independente da presença de HAS, os betabloqueadores são indicados para controle de frequência e ritmo cardíaco em fibrilação/flutter atrial e outras arritmias, como em arritmias supraventriculares, taquicardia sinusal e arritmias ventriculares específicas. ${ }^{9}$ Devido a maior prevalência de arritmias cardíacas e HAS com o envelhecimento, a classe pode ser uma opção inteligente para o tratamento de doenças coexistentes com um único fármaco.

\section{Gestação}

Os BBs são opções possíveis para o tratamento de HAS em gestantes ou em mulheres hipertensas que planejam engravidar, porém faltam estudos robustos que tragam maior segurança. O uso de IECA, BRA ou inibidores diretos da renina são contraindicados nessas ocasiões e os anti-hipertensivos de escolha são: metildopa, BCCs, hidralazina e os BBs, com exceção do atenolol, que associa-se a redução do crescimento fetal. ${ }^{17,18,39}$

\section{Tremor Essencial}

O tremor essencial é o distúrbio do movimento mais prevalente e seu risco aumenta significativamente com a idade. Sua prevalência entre idosos com 65 anos ou mais atinge cerca de $5 \%$ enquanto em nonagenários ultrapassa $20 \%{ }^{40}$ O propranolol foi o primeiro fármaco utilizado para o tratamento dessa condição e atualmente ainda é considerado a primeira escolha, exceto se contraindicações. Seu mecanismo de ação é incerto, mas acredita-se que se deva ao bloqueio de adrenoreceptores beta 2 periféricos. Outros BBs, como atenolol e metoprolol, também foram estudados e podem ser indicados para o tratamento, mas demonstraram menos resposta para essa finalidade. ${ }^{41}$ Pacientes hipertensos e portadores dessa condição podem se beneficiar do uso de BB em monoterapia para o tratamento concomitante dessas comorbidades.

\section{Enxaqueca}

Devido ao seu impacto na qualidade de vida, o tratamento profilático de crises de enxaqueca é fundamental. Trata-se de uma condição prevalente em aproximadamente $12 \%$ da população e sua fisiopatologia não é totalmente esclarecida. Acreditava-se que seu mecanismo era essencialmente vascular, mas atualmente sabe-se que outras vias estão envolvidas na sua fisiopatologia, dentre elas o sistema trigeminovascular, a ação serotoninérgica central, a sensibilização neuronal e a ação de peptídeos relacionados ao gene da calcitonina. ${ }^{42} \mathrm{O}$ uso de $\mathrm{BBs}$, em especial o propranolol, como tratamento profilático da enxaqueca é recomendado em diretrizes como primeira escolha e revisões sistemáticas sugerem que o fármaco pode reduzir sua frequência em até $50 \%{ }^{43}$ Não se sabe ao certo qual seu mecanismo de ação para reduzir a frequência de crises de enxaqueca, mas acredita-se que sua ação ocorra nos sistemas catecolamínicos e nos receptores serotoninérgicos centrais. Um estudo experimental em animais sugeriu que sua ação pode se relacionar com o antagonismo adrenérgico em receptores beta 1, inibindo o disparo de neurônios talamocorticais, modulando negativamente e talvez suprimindo a tendência à sensibilização central em neurônios de ordem superior. ${ }^{44}$

\section{BETABLOQUEADORES NA HIPERTENSÃO RESISTENTE}

O princípio básico do tratamento da hipertensão resistente é a associação de anti-hipertensivos que bloqueiem a maioria dos mecanismos fisiopatológicos de elevação da PA. Idealmente, deve ser prescrito um diurético, um bloqueador do sistema renina angiotensina e um bloqueador do canal de cálcio diidropiridínico, em doses plenas toleradas e a intervalos adequados.

A Diretriz da Sociedade Internacional de Hipertensão publicada em 2020, em acordo com as demais diretrizes vigentes, orienta o uso dos betabloqueadores como quinta opção no tratamento da hipertensão resistente. Porém, em situações particulares como DAC, ICC e taquiarritmias, um BB pode substituir o BCC no esquema terapêutico inicial com três medicações. ${ }^{17,18,34}$ 


\section{BETABLOQUEADORES NA EMERGÊNCIA HIPERTENSIVA}

Os principais BBs utilizados para a Emergência Hipertensiva estão listados no Quadro $4 .{ }^{18}$

\section{CONCLUSÃO}

Os betabloqueadores são utilizados há mais de 40 anos para o tratamento medicamento da hipertensão arterial. Existem diferenças importantes entre as moléculas, configurando uma classe heterogênea de anti-hipertensivos. Infelizmente as moléculas de terceira geração, com maior benefício potencial, não foram avaliadas em ensaios clínicos de hipertensão, havendo representação apenas de drogas clássicas, como o atenolol e o metoprolol, que demonstrou menor eficácia que outras classes. Faltam estudos que utilizem os BBs com propriedades vasodilatadoras e antioxidantes que demonstram melhor perfil metabólico e hemodinâmico, como o nebivolol e carvedilol.

No cenário atual e segundo as evidências científicas disponíveis hoje, os BB não são considerados fármacos de primeira linha no tratamento da HAS não complicada. Por sua vez, em situações especiais e em associação com outras condições clínicas, os BBs podem representar a melhor opção ou até mesmo ser considerados como terapêutica mandatória.

\section{REFERÊNCIAS}

1. Ahlquist RP. A study of the adrenotropic receptors. Am J Physiol. 1948;153(3):586-600. doi:10.1152/ajplegacy.1948.153.3.586

2. Powell CE, Slater IH. Blocking of inhibitory adrenergic receptors by a dichloro analog of isoproterenol. J Pharmacol Exp Ther. 1958;122(4):480-488.

3. Black JW, Stephenson JS. Pharmacology of a new adrenergic beta-receptorblocking compound (Nethalide). Lancet. 1962;2(7251):311-314. doi:10.1016/s0140-6736(62)90103-4

4. Black JW, Crowther AF, Shanks RG, Smith LH, Dornhorst AC. A new adrenergic betareceptor antagonist. Lancet. 1964;1(7342):1080-1081. doi:10.1016/s0140-6736(64)91275-9

5. Frishman WH. Beta-Adrenergic blockers: a 50-year historical perspective. Am J Ther. 2008;15(6):565-576. doi:10.1097/MJT.0b013e318188bdca

6. Frishman WH. Cardiovascular Pharmacotherapeutics. $3^{\mathfrak{a}}$ ed. Minnesota: Cardiotext Publishing; 2011.

7. Report of the Joint National Committee on Detection, Evaluation, and Treatment of High Blood Pressure. A cooperative study. JAMA. 1977;237(3):255-261. doi:10.1001/jama.1977.03270300059008

8. Ripley TL, Saseen JJ. $\beta$-blockers: a review of their pharmacological and physiological diversity in hypertension. Ann Pharmacother. 2014;48(6):723733. doi:10.1177/1060028013519591

9. López-Sendón J, Swedberg K, McMurray J, et al. Expert consensus document on beta-adrenergic receptor blockers. Eur Heart J. 2004;25(15):1341-1362. doi:10.1016/j.ehj.2004.06.002

10. Lund-Johansen P. Hemodynamic consequences of long-term beta-blocker therapy: a 5-year follow-up study of atenolol. J Cardiovasc Pharmacol. 1979;1(5):487-495. doi:10.1097/00005344-197909000-00001

11. Mangrella M, Rossi F, Fici F, Rossi F. Pharmacology of nebivolol. Pharmacol Res. 1998;38(6):419-431. doi:10.1006/phrs.1998.0387

12. Blumenfeld JD, SealeyJE, Mann SJ, et al. Beta-adrenergic receptor blockade as a therapeutic approach for suppressing the renin-angiotensin-aldosterone system in normotensive and hypertensive subjects. Am J Hypertens. 1999;12(5):451-459. doi:10.1016/s0895-7061(99)00005-9

13. Myers MG, Lewis PJ, Reid JL, Dollery CT. Brain concentration of propranolol in relation to hypotensive effect in the rabbit with observations on brain propranolol levels in man. J Pharmacol Exp Ther. 1975;192(2):327-335.
Quadro 4. Betabloqueadores parenterais para o tratamento das emergências hipertensivas.

\begin{tabular}{|c|c|c|}
\hline Medicação & $\begin{array}{c}\text { Modo de } \\
\text { administração e } \\
\text { dosagem }\end{array}$ & Indicação \\
\hline $\begin{array}{l}\text { Metoprolol } \\
\text { (BB seletivo) }\end{array}$ & $\begin{array}{l}5 \text { mg IV (repetir } \\
10 / 10 \text { min, se } \\
\text { necessário até } 20 \text { mg) }\end{array}$ & $\begin{array}{l}\text { Insuficiência } \\
\text { coronariana } \\
\text { Dissecção aguda de } \\
\text { aorta (em combinação } \\
\text { com NPS) }\end{array}$ \\
\hline $\begin{array}{l}\text { Esmolol } \\
\text { (BB seletivo de } \\
\text { ação ultra-rápida) }\end{array}$ & $\begin{array}{l}\text { Ataque: } 500 \mu \mathrm{g} / \mathrm{kg} \\
\text { Infusão intermitente } \\
25-50 \mu \mathrm{g} / \mathrm{kg} / \mathrm{min} \uparrow 25 \\
\mu \mathrm{g} / \mathrm{kg} / \mathrm{min} \text { cada } 10-20 \\
\text { min. } \\
\text { Máximo } 300 \mu \mathrm{g} / \mathrm{kg} / \\
\text { min }\end{array}$ & $\begin{array}{l}\text { Dissecção aguda de } \\
\text { aorta (em combinação } \\
\text { com NPS) } \\
\text { Hipertensão pós- } \\
\text { operatória grave }\end{array}$ \\
\hline $\begin{array}{l}\text { * Labetalol } \\
\text { (bloqueador } \mathrm{a} / \beta \text { - } \\
\text { adrenérgico) }\end{array}$ & $\begin{array}{l}\text { Ataque: } 20-80 \text { mg } \\
\text { 10-10 min } \\
\text { Infusão contínua } 2 \\
\text { mg/min (máximo } 300 \\
\text { mg/24h) }\end{array}$ & $\begin{array}{l}\text { AVE } \\
\text { Dissecção aguda de } \\
\text { aorta (em combinação } \\
\text { com NPS) }\end{array}$ \\
\hline
\end{tabular}

14. Gottschalk LA, Stone WN, Gleser GC. Peripheral versus central mechanisms accounting for antianxiety effects of propranolol. Psychosom Med. 1974;36(1):47-56. doi:10.1097/00006842-197401000-00004

15. Bengtsson K, Melander O, Orho-Melander M, et al. Polymorphism in the beta(1)-adrenergic receptor gene and hypertension. Circulation. 2001;104(2):187-190. doi:10.1161/01.cir.104.2.187

16. Bray MS, Krushkal J, Li L, et al. Positional genomic analysis identifies the beta(2)-adrenergic receptor gene as a susceptibility locus for human hypertension. Circulation. 2000;101(25):2877-2882. doi:10.1161/01. cir.101.25.2877

17. Malachias MVB, Gomes MAM, Nobre F, Alessi A, Feitosa AD, Coelho EB. 7ạ Diretriz Brasileira de Hipertensão Arterial: Capítulo 2 - Diagnóstico e Classificação.Arq Bras Cardiol. 2016;107(3 Suppl 3):7-13. doi:10.5935/ abc. 20160152

18. Williams B, Mancia G, Spiering W, et al. 2018 ESC/ESH Guidelines for the management of arterial hypertension. Eur HeartJ. 2018Sep 1;39(33):3021-3104. doi: 10.1093/eurheartj/ehy339. Erratum in: Eur Heart J. 2019 Feb 1;40(5):475. [published correction appears in Eur Heart J. 2019 Feb 1;40(5):475]. Eur Heart]. 2018;39(33):3021-3104. doi:10.1093/eurheartj/ehy339

19. Whelton PK, Carey RM, Aronow WS, et al. 2017 ACC/AHA/AAPA/ ABC/ACPM/AGS/APhA/ASH/ASPC/NMA/PCNA Guideline for the Prevention, Detection, Evaluation, and Management of High Blood Pressure in Adults: Executive Summary: A Report of the American College of Cardiology/American Heart Association Task Force on Clinical Practice Guidelines. Hypertension. 2018 Jun;71(6):1269-1324. doi: 10.1161/ HYP.0000000000000066. Epub 2017 Nov 13. Erratum in: Hypertension. 2018 Jun;71(6):e136-e139. Erratum in: Hypertension. 2018 Sep;72(3):e33. ACC/AHA/AAPA/ABC/ACPM/AGS/APhA/ASH/ASPC/NMA/PCNA Guideline for the Prevention, Detection, Evaluation, and Management of High Blood Pressure in Adults: A Report of the American College of Cardiology/American Heart Association Task Force on Clinical Practice Guidelines [published correction appears in J Am Coll Cardiol. 2018 May 15;71(19):2275-2279].J Am Coll Cardiol. 2018;71(19):e127-e248. Doi:10.1016/j.jacc.2017.11.006

20. Dahlöf B, Devereux RB, Kjeldsen SE, et al. Cardiovascular morbidity and mortality in the Losartan Intervention For Endpoint reduction in hypertension study (LIFE): a randomised trial against atenolol.Morbidade e mortalidade cardiovascular no estudo Losartan Intervention For Endpoint redução em hipertensão (LIFE): um ensaio randomizado contra atenolol. Lancet. 2002; 
359 (9311): 995-1003 doi: 10.1016/S0140-6736 (02) 08089-3

21. Dahlöf B, Sever PS, Poulter NR, et al. Prevention of cardiovascular events with an antihypertensive regimen of amlodipine adding perindopril as required versus atenolol adding bendroflumethiazide as required, in the Anglo-Scandinavian Cardiac Outcomes Trial-Blood Pressure Lowering Arm (ASCOT-BPLA): a multicentre randomised controlled trial. Lancet. 2005;366(9489):895-906. doi:10.1016/S0140-6736(05)67185-1

22. Carlberg B, Samuelsson O, Lindholm LH. Atenolol in hypertension: is it a wise choice? Lancet. 2004 Nov 6-12;364(9446):1684-9. doi: 10.1016/ S0140-6736(04)17355-8. Erratum in: Lancet. 2005 Feb 19;365(9460):656. Carlberg B, Samuelsson O, Lindholm LH. Atenolol in hypertension: is it a wise choice? [published correction appears in Lancet. 2005 Feb 19;365(9460):656].Lancet. 2004;364(9446):1684-1689. doi:10.1016/ S0140-6736(04)17355-8

23. Lindholm LH, Carlberg B, Samuelsson $\mathrm{O}$. Should beta blockers remain first choice in the treatment of primary hypertension? A meta-analysis. Lancet. 2005;366(9496):1545-1553. doi:10.1016/S0140-6736(05)67573-3

24. Bradley HA, Wiysonge CS, Volmink JA, Mayosi BM, Opie LH. How strong is the evidence for use of beta-blockers as first-line therapy for hypertension? Systematic review and meta-analysis. J Hypertens. 2006;24(11):2131-2141. doi:10.1097/01.hjh.0000249685.58370.28

25. Khan N, McAlister FA. Re-examining the efficacy of beta-blockers for the treatment of hypertension: a meta-analysis. CMAJ. 2006 Jun 6;174(12):1737-42. doi: 10.1503/cmaj.060110. Erratum in: CMAJ. 2007 Mar 27;176(7):976. [published correction appears in CMAJ. 2007 Mar 27;176(7):976].CMAJ. 2006;174(12):1737-1742. doi:10.1503/ cmaj.060110

26. Wiysonge CS, Bradley HA, Volmink J, Mayosi BM, Mbewu A, Opie LH. Beta-blockers for hypertension. Cochrane Database Syst Rev. 2012 Aug 15;(8):CD002003. doi: 10.1002/14651858.CD002003.pub3. Update in: Cochrane Database Syst Rev. 2012;11:CD002003. Cochrane Database Syst Rev. 2012;(8):CD002003. Published 2012 Aug 15. Doi:10.1002/14651858. CD002003.pub3

27. Elliott WJ, Childers WK. Should $\beta$ blockers no longer be considered first-line therapy for the treatment of essential hypertension without comorbidities? Curr Cardiol Rep. 2011;13(6):507-516. doi:10.1007/s11886-011-0216-z

28. Thomopoulos C, Parati G, Zanchetti A. Effects of blood pressure lowering on outcome incidence in hypertension: 4. Effects of various classes of antihypertensive drugs--overview and meta-analyses.J Hypertens. 2015;33(2):195-211. doi:10.1097/HJH.0000000000000447

29. Emdin CA, Rahimi K, Neal B, Callender T, Perkovic V, Patel A. Blood pressure lowering in type 2 diabetes: a systematic review and meta-analysis. JAMA. 2015;313(6):603-615. doi:10.1001/jama.2014.18574

30. Ettehad D, Emdin CA, Kiran A, et al. Blood pressure lowering for prevention of cardiovascular disease and death: a systematic review and meta-analysis. Lancet. 2016;387(10022):957-967. doi:10.1016/S0140-6736(15)01225-8

31. Thomopoulos C, Parati G, Zanchetti A. Effects of blood-pressure-lowering treatment on outcome incidence. 12. Effects in individuals with highnormal and normal blood pressure: overview and meta-analyses of randomized trials. J Hypertens. 2017;35(11):2150-2160. doi:10.1097/ HJH.0000000000001547

32. Dondo TB, Hall M, West RM, et al. $\beta$-Blockers and Mortality After Acute Myocardial Infarction in Patients Without Heart Failure or Ventricular Dysfunction. J Am Coll Cardiol. 2017;69(22):2710-2720. doi:10.1016/j. jacc.2017.03.578

33. Ziff OJ, Samra M, Howard JP, et al. Beta-blocker efficacy across different cardiovascular indications: an umbrella review and meta-analytic assessment. BMC Med. 2020;18(1):103. Published 2020 May 5. doi:10.1186/s12916-020-01564-3

34. Unger T, Borghi C, Charchar F, et al. 2020 International Society of Hypertension Global Hypertension Practice Guidelines. Hypertension. 2020;75(6):1334-1357. doi:10.1161/HYPERTENSIONAHA.120.15026

35. Yusuf S, Hawken S, Ounpuu S, et al. Effect of potentially modifiable risk factors associated with myocardial infarction in 52 countries (the INTERHEART study): case-control study. Lancet. 2004;364(9438):937-952. doi:10.1016/S0140-6736(04)17018-9

36. Law MR, Morris JK, Wald NJ. Use of blood pressure lowering drugs in the prevention of cardiovascular disease: meta-analysis of 147 randomised trials in the context of expectations from prospective epidemiological studies. BMJ. 2009;338:b1665. Published 2009 May 19. doi:10.1136/bmj.b1665

37. SPRINT Research Group, Wright JT Jr, Williamson JD, et al. A Randomized Trial of Intensive versus Standard Blood-Pressure Control. [N Engl J Med. 2015 Nov 26;373(22):2103-16. doi: 10.1056/NEJMoa1511939. Epub 2015 Nov 9. Erratum in: N Engl J Med. 2017 Dec 21;377(25):2506.published correction appears in N Engl J Med. 2017 Dec 21;377(25):2506].N Engl J Med. 2015;373(22):2103-2116. doi:10.1056/NEJMoa1511939

38. Beckett N, Peters R, Leonetti G, et al. Subgroup and per-protocol analyses from the Hypertension in the Very Elderly Trial. J Hypertens. 2014;32(7):1478-1487. doi:10.1097/HJH.0000000000000195

39. Abalos E, Duley L, Steyn DW, Gialdini C. Antihypertensive drug therapy for mild to moderate hypertension during pregnancy. Cochrane Database Syst Rev. 2018;10(10):CD002252. Published 2018 Oct 1. doi:10.1002/14651858.CD002252.pub4

40. Louis ED, Ferreira JJ. How common is the most common adult movement disorder? Update on the worldwide prevalence of essential tremor. Mov Disord. 2010;25(5):534-541. doi:10.1002/mds.22838

41. Hedera P, Cibulčík F, Davis TL. Pharmacotherapy of essential tremor. J Cent Nerv Syst Dis. 2013;5:43-55. Published 2013 Dec 22. ddoi:10.4137/ JCNSD.S6561

42. Cutrer FM. Pathophysiology of migraine. Semin Neurol. 2006;26(2):171180. doi:10.1055/s-2006-939917

43. Jackson JL, Kuriyama A, Kuwatsuka Y, et al Beta-blockers for the prevention of headache in adults, a systematic review and meta-analysis. PLoS One. 2019;14(3):e0212785. Published 2019 Mar 20. doi:10.1371/journal. pone.0212785

44. Shields KG, Goadsby PJ. O propranolol modula as respostas trigeminovasculares no núcleo ventroposteromedial do tálamo: um papel na enxaqueca ?. Brain . 2005; 128 (Pt 1): 86-97. doi: 10.1093/brain/awh298. 